Volume 8, No.6, November-December 2019

International Journal of Science and Applied Information Technology

Available Online at http://www.warse.org/ijsait/static/pdf/file/ijsait19862019.pdf

https://doi.org/10.30534/ijsait/2019/198620198

\title{
Wireless Power Communications and Natural Language processing in multi Radio deployment
}

\author{
Belal Abuhaija \\ Department of Mobile Computing, Amman Arab University, Jordan. \\ balhaijaaau@aau.edu.jo
}

\begin{abstract}
Deployment of new radio technologies in the mobile world has been gaining momentum as an alternative to meet the ever-increasing demands of data customers. This issue is motivated by the existence of applications, which make available to ordinary users, a host of services that require higher spectral efficiency and data rates. The deployment of LTE concurrently with the existing legacy cellular systems, such as UMTS in the same cell sites, has proven to enhance the network resources available to the operators in the form of common radio resource management (CRRM). We aim to develop an algorithm based on several criteria to enhance the decision making process of the operators' network and to minimize sudden changes in heterogeneous cell loads in order to optimize the overall system capacity, efficiency, Language used and power consumption in Cellular Networks.
\end{abstract}

Key words : Energy Consumption, LTE, Radio Resources, $3 \mathrm{G}$.

\section{INTRODUCTION}

Rapid evolution of wireless communication systems coupled with the spread of handheld devices such as smartphones and tablets, along with their associated power-hungry applications, accounts for the consumption of the majority of battery energy. A new paradigm in research that aims at prolonging the battery life of such devices has emerged in the form of wireless energy harvesting. As a result, research has focused on methods to maximize spectral efficiency by deriving an optimal time-split factor between energy harvesting, information processing, and transmission.

Many challenges and opportunities can be identified; among others, mobility, load balancing and traffic steering. Heterogeneous applications coupled with heterogeneous access technologies make the management of radio access selection rather complex, adding to that, the limited energy of the handheld devices. Therefore, in this research, we propose a multi-criterion cell selection algorithm for users in idle mode as well as in connected mode. Another aim is to design an algorithm for user steering and traffic offloading. The Proposed algorithms include criterion such as multi radio environment based on service request, user profile history and radio measurements.

Another aim is to design an optimal time-split factor between energy harvesting and information transmission/reception is derived in the case of full-duplex transmission for symmetric traffic and asymmetric traffic. Second, a closed-form mathematical expression is derived for delay constraint traffic as well as elastic traffic services. Third, the constraints imposed by traffic profiles were considered. Hence, mathematical expression tends itself to realistic and practical analysis of long-term evolution (LTE) systems including SINR. Deployment of new radio technologies in the mobile world has been steadily gaining momentum as an alternative to meet the ever increasing demands of customers. This has especially been motivated by the existence of applications that make available to ordinary users a host of services that require higher spectral efficiency and data rates. LTE (Long Term Evolution) and LTE Advanced (LTE-A) with an enhanced air interface and optimized packet data architecture, an all IP network, is envisioned to provide enhanced data rates, reduced latency and cost efficient operations. The deployment of LTE concurrently with the existing legacy cellular systems, such as UMTS in the same cell sites, has proven to enhance the network resources available to the operators. Such deployment, however, is accompanied with many challenges such as mobility and load balancing. The diversity and availability of services which can be supported by several radio access networks, such as legacy $3 \mathrm{G}$ and LTE, make the management of radio access selection rather complex. In this paper, we propose a multi-criterion cell selection algorithm for multi radio environment ( $3 \mathrm{G}$ and LTE) based on service request, user profile and user equipment capabilities [1-5].

To investigate and identify the challenges in the path of LTE system performance evaluation to reach an optimum solution for such challenges. In evaluating LTE System, we are faced with many challenges of obtaining suitable and accurate results. The variety of entities and stakeholders involved, the quality of service (QoS) requested through the required data rates as well as the instantaneous propagation conditions can 
influence the system capacity a great deal. Such challenges are further complicated by the presence of multiple technologies in the same cell site such as $(2 \mathrm{G}, 3 \mathrm{G}, \mathrm{ETC} \ldots)$. As such, the common radio resources scheduling (CRRM) becomes a focal point of investigation [5-10].

A new paradigm has been emerged in the research aiming at prolonging the battery life of such devices has emerged in the form of wireless energy harvesting. As a result, research has focused on ways and means to maximize spectral efficiency by deriving an optimal time split between energy harvesting and information processing and transmission. However, this was done without considering LTE resources scheduling, traffic profiles, and quality of service (QoS). In this paper, first, an optimal time split between energy harvesting and information transmission/ reception is derived in the case of full duplex transmission for symmetric traffic and a symmetric traffic, second, a closed form mathematical expression is derived for delay constraint traffic as well as elastic traffic services, third, the constraints imposed by traffic profiles were considered, hence, mathematical expression tends itself to realistic and practical analysis of LTE systems evidenced by the results presented. We consider a source powered by a dedicated PBS in a point to point communication link. The time splitting approach is realized between the harvested energy from the power source and the time to perform information transmission/reception with the harvested power. Therefore, and in order to understand the limitations of wireless powered communication links, we analyze the achievable average throughput of elastic applications such as file transfer and delay constraint applications such as VoIP applications in a full duplex communication LTE system. The optimal time splitting between the power harvesting and information transmission which maximizes the average throughput is investigated.

Numerical and simulation results have shown that increasing the number of antennas at the PBS can significantly improve the system throughput to a certain degree. This is consistent with the notion that the larger number of antennas can provide multi path of energy to the mobile station, and hence yields better energy efficiency. It is also shown that the time split is a key parameter in determining the performance of the LTE system while considering full duplex transmission while accounting for the constraints imposed by the traffic profiles. This research shall be extended to include natural language processing in correlation with wireless network architecture; two problems in particular make processing of natural languages difficult and cause different techniques to be used than those associated with the construction of compilers for processing artificial languages. Problems such as, first, the level of ambiguity that exists in natural languages, second, the complexity of semantic information contained in even simple sentences. Typically, language processors deal with large numbers of words, many of which have alternative uses, and large grammars which allow different phrase types to be formed from the same string of words. Language processors are made more complex because of the irregularity of language and the different kinds of ambiguity which can occur. Many researchers explore this topic and still more work needs to be done as this is an ongoing problem. Most recently and in Arabic language, the including of slang Arabic language has been explored. New and interesting results shall be investigated.

\section{LITRATURE REVIEW}

The LTE architecture is simplified to become a more efficient all-IP system that is optimized for packet traffic. For example, Radio Network Controller (RNC) used in early 3G releases for Radio Resource Management (RRM) functions is removed and its intelligence is moved to the Evolved Node B (eNodeB). Another considerable difference to legacy cellular systems is that there is no circuit switched domain in LTE architecture. The core network is solely all-IP, and therefore control data and user data as well as voice are all transferred on top of packet switched IP-protocol. Therefore, a fundamental change to the mobility architecture and services has been introduced when compared to the legacy 3GPP technologies. UMTS has a radio network controlling element (Radio Network Controller) which possesses the necessary intelligence and signaling capabilities. Coexistence of LTE and UMTS/HSPA radio technologies is the most likely scenario in the near future when the same operator is deploying the new LTE RAT in the same cell site of UMTS/HSPA.

Devising a mechanism for seamless internetworking between LTE and UMTS/HSPA is an essential step to facilitate multi RAT deployment. Inter operation between LTE and UMTS can enhance the radio resources of the operator as each of the internetworking technologies, LTE and UMTS/HSPA RATs, has its own radio resources. The collection of these radio resources is forming one big radio resource pool under the administration of one multi-standard radio (MSR) base station. Such MSR base station is characterized by the ability of its receiver and transmitter to process two or more carriers in common active RF components simultaneously in a declared RF bandwidth, where at least one carrier is of a different RAT than the other carrier(s).

For efficient utilization of the radio resource pool, the operator needs to devise advanced management algorithm for call redirection and user relocation. Such approach can have a positive impact on the network resource utilization and reduce the resource burden on the core network and at the same time enhance mobility and load balancing. Internetworking between LTE and UMTS/HSPA can improve load balancing among RATs depending on the deployment scenarios as envisioned in the standard [10]. Currently the load balancing handover is based on the resource usage in each cell. However, resource usage does not 
indicate the level of user satisfaction by the service or what is called "better user experience". At the same time, such problem is complicated by other issues, such as; the diversity of the services, the load information exchanging between UMTS and LTE via Radio Access Network Information Management (RIM-PDU). In this paper, we are presenting an algorithm for load balancing based on service request by the user and UE capabilities. The signaling problem due to inter RAT communication is not considered. The scenario we are basing this algorithm on is full coverage scenario.

With the deployment of many radio technologies and the planned wide installation of such radio technologies in the same cell site and by the same operator; the need for load self-balancing networks has been on the rise for the last few years. The starting point was the introduction of Self Organizing Network (SON) by the 3GPP community $[12,13]$. One of our aims in this research is to develop an algorithm based on several criteria to enhance the decision making process of the operators' network and to minimize sudden changes in heterogeneous cell loads in order to optimize the overall system capacity and efficiency. In [14], the authors proposed two fuzzy models for load balancing. Besides the key performance indicators, the two models use load indicators as well for a more accurate load balancing. The virtual cell load was computed based on the number of users in the cell compare to the number of resources used. The number of unsatisfied customers was measured based on the cell virtual load and the number of customers in the cell. The authors developed two models, the Load distribution index and the unsatisfied user model. For load distribution index, the virtual cell load and the overall system load are used as input parameters. The number of unsatisfied users is also used as an input parameter along with the virtual load and overall system load as input parameters. The authors are emphasizing the need for human intuition to solve such nonlinear problem. In [7], the authors proposed to use fuzzy logic controller (FCL) for handover parameters optimization. The controller makes use of the reference symbol received power of the serving cell and the adjacent cell. The second parameter used is the load of the cells based on the mean number of time slots occupied, while the third parameter is the current value of handover margin. The authors claim that the call blocking ratio has been improved by using FCL for self-optimizing handover margins in LTE networks. In [heterogeneous service], the authors formulated the load balancing problem as multi objective optimization problem based on heterogeneous services in LTE. Two classes of service have been considered, constant bit rate and best effort services. The constant bit rate users are assigned the required resources first and then the residual resources are assigned to the best effort users. During admission control, the user with constant bit rate takes priority if resources are available. This is an indication that the constant bit rate users are taking priority over the best effort users and subject to the total resources available in the system. The authors claim that the proposed load balancing algorithm can enhance the blocking probability of the constant bit rate services and enhance the best effort services throughput.

The models and algorithms presented in the above research and within references, to provide a solution for self-organizing networks are limited to a single deployment of one technology; LTE technology. However, the future of cellular systems deployment is to deploy multiple radio technologies (RATs) in the same cell site. Moreover, an efficient algorithm for an efficient cell reselection can enhance greatly the load balancing of the overall system and reduce the penalty of signaling due to cell reselection and handover.

In recent years and due to the wide spread of smart phones and handheld internet access devices along with the availability of high data rate multimedia traffic, energy consumption has been exponentially increasing both at the base station as well as the access devices. However, while the base stations are continuously charged by the power grid, the access devices battery power limited and in need of frequent recharging.

One of the promising techniques to address the power limitation of the access devices is to employ radio frequency signal based energy harvesting [8-13].

RF signals can carry both energy and information; a new research area has emerged to make use of such characteristic of the RF signal and is called simultaneous wireless and power transfer (SWIPT). In [8], the authors proposed a real deployment of an energy harvesting architecture, where the optimal rate energy region is established. In [13] and [14] the authors have investigated the basic tradeoff between system capacity and energy for SWIPT system by characterizing the communication systems with large received energy per unit time and large information per unit time simultaneously. In [8], the authors investigated the rate energy region considering imperfect channel state information (CSI).

In [12], based on orthogonal frequency division multiple access (OFDMA) systems the authors studied a resource allocation algorithm to maximize the energy efficiency of data transmission. The authors derived an algorithm that includes the minimum data rate for the delay constraint services and the data rate of the system, and the minimum amount of power needed to be delivered to the receivers. In [16], amplify-and-forward (AF) relaying system throughput performance was studied for time switching and power splitting protocol. The received RF signal is used by the relay node to harvest energy; then, the relay node uses the harvested energy to forward the information to the destination. An expression for the outage probability has been derived to determine the system throughput for delay limited and the ergodic capacity has been derived for delay tolerant services. In [17], a power allocation strategy for decode-and-forward (DF) relaying system with multiple source-destination pairs is 
studied. A relay is harvesting energy and distributing the energy among the current users in the system, which is used by the different users for uplink information transmission.

In general researchers have limited their studies to the integrated base station architecture that provides both information and energy source. The gap between the needed energy to transmit information and the harvested energy is large. The sensitivity difference between the two required powers is in the order of $\log (100 \mathrm{dBm} / 10 \mathrm{dBm})$. Therefore, the mobile devices need to be in the range of 10 meters or less from the base station in order to deliver meaningful energy [18]. As such, the deployment of the integrated base stations is in high dense areas only, which is deemed to be impractical due to the huge operational cost.

In [10], the authors proposed an architecture to separate the information source from the power source. They proposed a power beacon station (PBS) to be deployed in dense areas and separate from the base station which handles the transmission of information. The relationship between the PBS and base station densities was outlined under the outage probability constraint. Such design is deemed to be practical and cost effective to ensure the deployment of SWIPT in practical scenarios. Most of the above research did not consider that telecommunication systems are full duplex and the type of service, i.e. delay tolerant services and delay constraint services.

The delay requirements of the service impose a certain restriction on the practicality of time split. On the other hand, elastic service such as FTP, uplink requests have minimal effect on power consumption while the downlink data rate has the maximum effect [19-21].

In this part of the research we shall adopt a close paradigm to the one presented in [10], except that we realize LTE systems and all the devices part of the system are equipped with multiple antennas. We also develop the solutions to include the internet traffic profiles. We consider VoIP and FTP services. Both the PBS and the base station are both operating at different frequencies. At the same time, we consider that each MS is equipped with multiple antennas for Energy harvesting and multiple antennas for information sending and receiving to ensure full MIMO on both aspects of the system [8].

\section{CONCLUSION}

With the introduction of next generation networks $5 \mathrm{G}$ and others, services still play a major role in deployment and power consumption. Wireless power transfer is one of the solutions to combat the device battery consumption. Energy consumption depends not only on the application but also on the language used (English or Arabic). Therefore, new studies should be done on languages other than English. Many challenges are presented in this paper and a wide range of research ideas to be investigated in the future.

\section{REFERENCES}

1. 3rd Generation Partnership Project (3GPP), Technical Specification Group Services and System Aspects; Service Aspects, Service and Service Capabilities, 3GPP TS 22.105 V11.0.1 (2012-11), Release 11.

2. 3rd Technical Specification Group Services and System Aspects; General Packet Radio Service (GPRS) enhancements for Evolved Universal Terrestrial Radio Access Network (E-UTRAN) access (Release 12), 3GPP TS 23.401 V12.3.0 (2013-12).

3. 3rd Generation Partnership Project; Technical Specification Group Radio Access Network; RAN enhancements for UMTS/HSPA and LTE interworking (Release 12) 3GPP TR 37.852 V12.0.0 (2013-12).

4. 3rd Generation Partnership Project; Technical Specification Group Services and System Aspects; Telecommunication Management; Self Organizing Networks (SON); Concepts and requirements (Release 11). 3GPP TS 32.500 V11.1.0 (2011-12).

5. 3rd Generation Partnership Project; Telecommunication management; Self-Organizing Networks (SON) Policy Network Resource Model (NRM) Integration Reference Point (IRP); Requirements, 3GPP TS 32.521 V11.1.0 (2012).

6. Aderemi A. Atayero, Matthew K. Luka, Adeyemi A. Alatishe," Neural-encoded Fuzzy Models for Load Balancing in 3GPP LTE" International Journal of Applied Information Systems, Volume 4 Number 1, 2012.

https://doi.org/10.5120/ijais12-450628

7. J. Rodriguez, de la Bandera, I.;Munoz, P. ;Barco, R. "Load Balancing in a Realistic Urban Scenario for LTE Networks", Vehicular Technology Conference (VTC Spring), Budabest, 2011 IEEE 73rd.

8. $\mathrm{R}$ Zhang, CK Ho, MIMO broadcasting for simultaneous wireless information and power transfer, IEEE Transactions on Wireless Communications, Volume: 12, Issue: 5, May 2013.

9. Xun Zhou; Rui Zhang; Chin Keong Ho, Wireless Information and Power Transfer: Architecture Design and Rate-Energy Tradeoff, IEEE Transactions on Communications, Volume: 61, Issue: 11, November 2013.

10. Kaibin Huang; Vincent K. N. Lau, Enabling Wireless Power Transfer in Cellular Networks: Architecture, Modeling and Deployment, IEEE Transactions on Wireless Communications, Volume: 13, Issue: 2, February 2014.

11. Liang Liu; Rui Zhang; Kee-Chaing Chua, Wireless Information and Power Transfer: A Dynamic Power Splitting Approach, IEEE Transactions on Communications, Volume: 61, Issue: 9, September 2013.

12. Derrick Wing Kwan Ng; Ernest S. Lo; Robert Schober, Wireless Information and Power Transfer: Energy 
Efficiency Optimization in OFDMA Systems, IEEE Transactions on Wireless Communications, Volume: 12, Issue: 12, December 2013.

13. Lav R. Varshney, Transporting information and energy simultaneously, Information Theory, ISIT, 2008.

14. [14] P Grover, A Sahai, Shannon meets Tesla: Wireless information and power transfer, ISIT, 2010.

15. Zhengzheng Xiang; Meixia Tao, Robust Beamforming for Wireless Information and Power Transmission, IEEE Wireless Communications Letters, Volume: 1, Issue: 4, August 2012.

16. [16] Ali A. Nasir; Xiangyun Zhou; Salman Durrani; Rodney A. Kennedy, Relaying Protocols for Wireless Energy Harvesting and Information Processing, IEEE Transactions on Wireless Communications, Volume: 12, Issue: 7, July 2013.

17. Z Ding, SM Perlaza, I Esnaola, Power allocation strategies in energy harvesting wireless cooperative networks, IEEE Transactions on Wireless Communications, Volume: 13, Issue: 2, February 2014.

18. Kaibin Huang; Xiangyun Zhou, Cutting the last wires for mobile communications by microwave power transfer, IEEE Communications Magazine, Volume: 53, Issue: 6, June 2015. https://doi.org/10.1109/MCOM.2015.7120022

19. Junxian Huang, Feng Qian, Alexandre Gerber, Z. Morley Mao, Subhabrata Sen, Oliver Spatscheck, A close examination of performance and power characteristics of 4G LTE networks, Proceeding MobiSys, Pages 225-238, UK, June 25 - 29, 2012.

20. Anders R. Jensen ; Mads Lauridsen ; Preben Mogensen ; Troels B. Sørensen ; Per Jensen, LTE UE Power Consumption Model: For System Level Energy and Performance Optimization, IEEE Vehicular Technology Conference (VTC Fall), 2012.

21. Bjoern Dusza; Christoph Ide; Liang Cheng; Christian Wietfeld, An accurate measurement-based power consumption model for LTE uplink transmissions, IEEE Computer Communications Workshops (INFOCOM WKSHPS), 2013.

https://doi.org/10.1109/INFCOMW.2013.6970731 